\title{
High-Pressure Gas Phase Femtosecond Laser Ionization Mass Spectrometry
}

\author{
Jiahui Peng, ${ }^{\dagger}$ Noah Puskas, ${ }^{\dagger}$ Paul B. Corkum, ${ }^{\dagger,}$ David M. Rayner, ${ }^{*}$, and Alexandre V. Loboda $^{*}$, \\ ${ }^{\dagger}$ University of Ottawa, Ottawa, Canada, K1N 6N5 \\ ${ }^{ \pm}$National Research Council of Canada, Ottawa, Canada, K1A 0R6 \\ ${ }^{\S}$ AB SCIEX, 71 Four Valley Drive, Concord, Ontario, Canada \\ Supporting Information
}

ABSTRACT: We describe a novel ion source for analytical mass spectrometry based on femtosecond laser ionization at pressures at and above atmospheric and characterize its performance when coupled to a tandem quadrupole/time-of-flight mass spectrometer. We assess source saturation limits, ionization and sampling efficiencies, the effective ionization volume, and limits of detection. We demonstrate $100 \%$ efficient ionization for a set of organic compounds and show that the degree of ion fragmentation over a range of laser powers is favorable compared to electron impact ionization, especially in that a substantial parent ion signal is always observed. We show how collisional cooling plays a role in

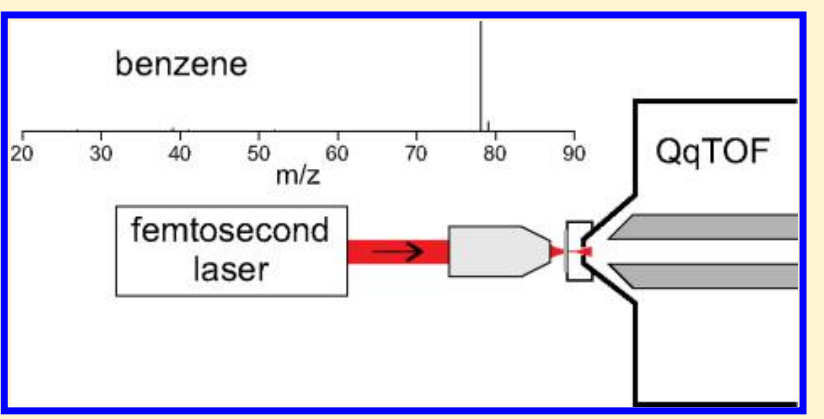
controlling fragmentation at high pressures and address how ion-molecule chemistry can be controlled or exploited. Highpressure femtosecond laser ionization will allow "universal" and efficient ionization, presenting a research direction that will broaden the options for gas phase analysis beyond the capabilities of electron impact ionization.

$\mathrm{I}_{\mathrm{m}}^{\mathrm{o}}$ onization methods play an important role in the advances of modern mass spectrometry. The development of electrospray ionization (ESI) and matrix-assisted laser desorption/ ionization (MALDI) ${ }^{1}$ in the late 1980s significantly broadened the range of organic compounds that can be analyzed by mass spectrometry. ESI and MALDI enabled ionization of large organic ions with good efficiency and without significant fragmentation. A variety of ionization techniques continued to build upon the ESI and MALDI principles including desorption electrospray ionization (DESI), ${ }^{2}$ orthogonal-injection MALDI TOF with collisional cooling, ${ }^{3}$ and laser ablation ESI. ${ }^{4}$ As a result, many ionization methods are available today for analysis of samples presented as dilutions in the liquid or solid form.

In contrast, analysis of samples in the gas phase is still dominated by electron impact (EI) ionization. Despite its limitations, such as a relatively low ionization efficiency and a significant degree of fragmentation for large organic molecules, EI is considered the gold standard of gas phase ionization. Among many factors that make EI popular is "universality" of ionization. In other words, virtually any molecule present in the gas phase will provide an ion signal, albeit in some cases the bulk of the signal is concentrated in the fragment ions.

On the basis of studies in high vacuum, nonresonant strongfield ionization of organic molecules with intense femtosecond pulses has been proposed as a "universal" ionization method with moderate ion fragmentation and ionization probabilities that reach unity. ${ }^{5}$ It is referred to as field ionization because the underlying physics is shared with static field ionization as employed in field ionization mass spectrometry. ${ }^{6}$ It is universal because the tunnel ionization probability of atoms or molecules to this intense field process depends, to a large extent, only on the ionization potential. ${ }^{7,8}$ Because of its nonresonant nature and unlike single photon UV ionization and resonant multiphoton ionization, it is not sensitive to molecular structure. This means the technique does not require tuning the laser wavelength to a molecule specific resonance.

Two factors that have limited adoption of femtosecond laser ionization (fsLI) in mass spectrometry are the complexity of femtosecond lasers and the limited size of the ionization volume, as determined by the need to focus the laser to achieve the necessary intensity (energy flux) for nonresonant ionization. The former is being solved by the relentless progress of laser technology. We demonstrate that the latter can be solved by ionizing at pressures at and above atmospheric pressure. By increasing the pressure, one compresses a larger number of analyte molecules into the same volume, a feature that is extremely valuable when operating with a limited ionization volume. We report on the development of highpressure femtosecond laser ionization (HP-fsLI) as a mass spectrometer ion source for gas phase analysis.

We implement HP-fsLI by modifying an atmospheric pressure sampling mass spectrometer designed for electrospray operation. We characterize HP-fsLI using the ionization of xenon as a test case to understand limits placed by source

Received: March 15, 2012

Accepted: May 30, 2012

Published: May 30, 2012 
saturation, establish that ion yields scale directly with laser repetition rate and with analyte concentration, show that ion yields can be modeled quantitatively, demonstrate $100 \%$ ionization efficiency, and assess overall ion detection efficiencies and detection limits. We then discuss the HP-fsLI of three organic molecules chosen to represent three classes of compounds that exhibit different fragmentation behavior under low pressure fsLI. We confirm that $100 \%$ efficient ionization of all three classes can be achieved by HP-fsLI. We address issues of fragmentation, which is found to be generally favorable compared to EI, and the role that ion-molecule chemistry can play at high pressure. Finally we develop a simplified model for predicting ionization yields that provides a prescription for optimizing the irradiation conditions and also demonstrates that the focal geometry makes the performance of HP-fsLI insensitive to the peak laser intensity as long as the intensity passes the threshold intensity where ionization probably grows rapidly toward $100 \%$.

\section{EXPERIMENTAL SECTION}

The experiments were carried out using a modified QStarXL (AB Sciex, Concord, Canada) mass spectrometer. The QStarXL is a quadrupole/time-of-flight (QqTOF) MS/MS instrument with an electrospray ionization capability that lends it to adaption for high-pressure ionization studies. QqTOF spectrometers have the capacity to operate with pulsed ion sources by converting a pulsed ion beam into a quasicontinuous beam. ${ }^{3}$ We replaced the electrospray front-end of the instrument with the custom built fsLI interface shown schematically in Figure 1. Ionization takes place at the focus

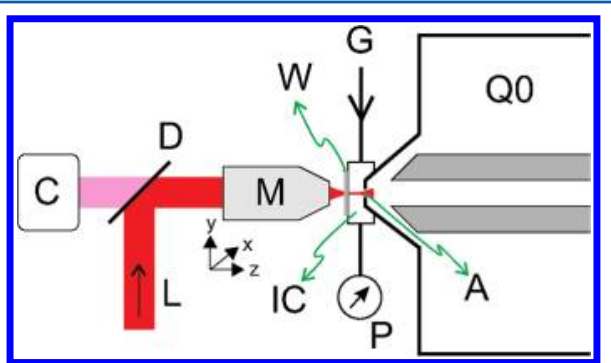

Figure 1. Schematic of the experimental setup for femtosecond laser ionization at high pressure. The ionization cell, IC, is connected to the mass spectrometer quadrupole ion guide inlet chamber, Q0, by a small aperture, $\mathrm{A}$. The femtosecond laser beam, $\mathrm{L}$, is reflected off a dichroic mirror, $\mathrm{D}$, and focused in IC through a window, $\mathrm{W}$, using a microscope objective, $M$, mounted on an $x y z$ translation stage. The window has a transparent conductive coating on the inside surface that can be biased to push ions toward the aperture. Gas enters IC through an inlet G, and the pressure, maintained by balancing the inlet flow with the pumping rate through the aperture, is measured by gauge $\mathrm{P}$. A camera, C, setup to view the aperture through the mirror, $\mathrm{D}$, is used to assist laser alignment.

of the femtosecond laser directly in front of a small aperture into the quadrupole ion guide inlet chamber, Q0, of the mass spectrometer. Because nonresonant femtosecond laser ionization requires intense fields, ionization is confined to the focal region. Ions are pulled into $\mathrm{Q} 0$ by the flow of gas driven by the pressure differential between the ionization cell, IC, and Q0, assisted by a voltage applied to a transparent conducting coating on the inner surface of the window through which the laser beam is introduced. The position of the laser focus in the cell is adjusted by moving the microscope objective, used to focus the laser beam, on an $x y z$ translation stage. Care must be taken when aligning the laser beam to avoid focusing the laser in the window or laser machining the aperture. We use a camera, C, setup to view the aperture through a dichroic mirror, to help make the alignment. Once aligned, the ion source is robust to laser damage as long as the focus is only moved on the $z$-axis and the focus is not brought so close to the window that it experiences intensities greater than a conservative limit of $10^{12} \mathrm{~W} \mathrm{~cm}{ }^{-2}$. With our present cell dimensions, this condition gives us a usable range of $>1.1 \mathrm{~mm}$ in $z$ using a 0.1 NA objective.

The enclosed ionization cell performs two functions. First, it allows gas flows to be studied. Second it allows the use of a carrier gas other than air. With intense field laser ionization at high pressure, the limiting background, in the absence of contaminants, is determined by ionization of the carrier or background gas. As demonstrated in the Supporting Information, $\mathrm{N}_{2}$ and air generally ionize too easily to give acceptable discrimination for organics.

To study organic molecules, where fragmentation can be an issue, we also found it necessary to replace the nitrogen gas, used in the standard QStar configuration as buffer gas in the entrance quadrupole ion guide chamber (Q0) and in the collision cell (Q2), with $\mathrm{He}$. We determined in MS/MS experiments that certain organic ions showed significant collisional fragmentation in Q2 even at collisional energies as low as $5 \mathrm{~V}$ when $\mathrm{N}_{2}$ was used. As $5 \mathrm{~V}$ is the limit below which ions are not transmitted efficiently to the TOF chamber, we changed the buffer gas to He. With this less efficient collision gas we were able to reduce collision induced fragmentation in the MS itself to an insignificant level.

When the system is run fully on $\mathrm{He}$ (ion source and MS buffer gas), He consumption is $150 \mathrm{sccm}$, mainly as MS buffer gas. If collisional fragmentation in the MS is not an issue (this depends on the analytes under study) $\mathrm{N}_{2}$ can be used as the buffer gas and He consumption is reduced to that of the ion source. The flow through the ion source is determined by the pressure in the source and the aperture size. With the source at $1 \mathrm{~atm}$ and a $40 \mu \mathrm{m}$ diameter aperture the flow is $15 \mathrm{sccm}$. We have yet to test the source with a smaller aperture but expect no problems at least down to $10 \mu \mathrm{m}$ diameter where the flow rate would be $1 \mathrm{sccm}$.

Gas mixtures are prepared in a storage bottle attached to a gas manifold. Low concentrations of analyte are obtained by successive dilutions of a stock mixture made using a capacitance manometer (MKS Baratron 627B) to measure the initial partial pressure of the analyte. Flow into the ionization cell is controlled by an in-line regulator followed by a length of flow restricting capillary tubing. The pressure in the cell is measured using a silicon pressure transducer (Omega PX309).

The Ti:Sapphire laser system operates at a wavelength of 800 $\mathrm{nm}$ and produces pulses of $40 \mathrm{fs}$ and longer at energies up to 3 $\mu \mathrm{J}$ and at repetition rates from single shot to $100 \mathrm{kHz}$. It consists of a Kerr lens mode-locked femtosecond oscillator (KMlabs) and an NRC built chirped pulse amplification system including a regenerative amplifier (RegA, Coherent). The pulse duration is controlled at the experiment (between 40 fs and 1.5 ps) by applying a quadratic chirp through changing the gratingmirror separation in the compressor. Prechirping is used to compensate for dispersion in the optical path between the laser and the ionization cell. The repetition rate of the laser is controlled through the external triggering of the RegA cavity Qswitch. The trigger signal is provided by a master clock and can 
be varied between the single shot and $100 \mathrm{kHz}$. The laser beam is spatially filtered at the output of the pulse compressor using a telescope with a pinhole to ensure a high-quality Gaussian spatial mode. The laser is focused with either a $0.1 \mathrm{NA}$ (magnification $4 \times$ ) or a 0.25 NA (magnification 10X) microscope objective obtained from Edmunds Optics. These specifications refer to their use in a DIN standard microscope. When used to focus the collimated laser beam with their apertures fully filled, their measured working distances are 6.4 and $2.0 \mathrm{~mm}$, respectively. The pulse energy is adjusted using a half-wave-plate/polarizer combination in the beam that can be adjusted under computer control when performing intensity studies. The pulse energy was calculated from the laser power measured before the microscope objective by a power meter (Thorlabs PM-30) and was corrected for the independently measured transmission of the microscope objective and window.

\section{SAFETY CONSIDERATIONS}

The laser used in these studies presents the possibility of ocular damage through direct exposure to the beam. The work described in this paper was carried out in a research laser facility following laser safety practices and policies of the National Research Council of Canada and University of Ottawa. The development of HP-fsLI MS for nonlaser specialists will require attention to laser safety issues by using strategies such as laser beam confinement.

\section{RESULTS AND DISCUSSION}

Ionization Efficiency. Our first set of HP-fsLI experiments was directed at demonstrating $100 \%$ ionization efficiency, determining the effective ionization volume and the ion collection efficiency of the setup, and identifying issues presented by operating at high pressure. We chose Xe as the analyte for these calibration experiments for several reasons: (1) The intense field ionization of Xe has been extensively studied under collision free conditions in high vacuum. Xe ionization rates are well reproduced by PPT theory; ${ }^{9}$ (2) As an atom, $\mathrm{Xe}$ has no complication from fragmentation when measuring ion yields; and (3) Xe has a distinctive natural isotope distribution. This helps in analyzing saturation effects. In addition, the low-abundance isotopes can be used to establish limits of detection (LOD) without extreme dilution.

To determine the dynamic range of the MS system and to establish conditions for reliable quantitative measurements, it is important to understand saturation limits and to distinguish those limits intrinsic to the MS from those imposed by the HPfsLI process itself. Ion counting systems have pulse pile-up limitations that put a limit on the count rate for individual peaks. As detailed in the Supporting Information, measurement of apparent $\mathrm{Xe}$ isotope distributions sets the single peak pile-up saturation limit at $1.25 \times 10^{5}$ counts/s in our experiments. A second saturation effect intrinsic to the MS involves overloading the data transfer channel from the time-to-digital converter (TDC) to the acquisition computer. For the QStarXL this puts a limit of $\sim 10^{6}$ counts/s on the total ion count.

The occurrence of source saturation directly associated with HP-fsLI is apparent from Figure 2a. The figure plots the laser pulse energy dependence of the normalized measured $\mathrm{Xe}$ ionization signal at different concentrations of Xe in He. In the absence of source saturation, these curves should be super-

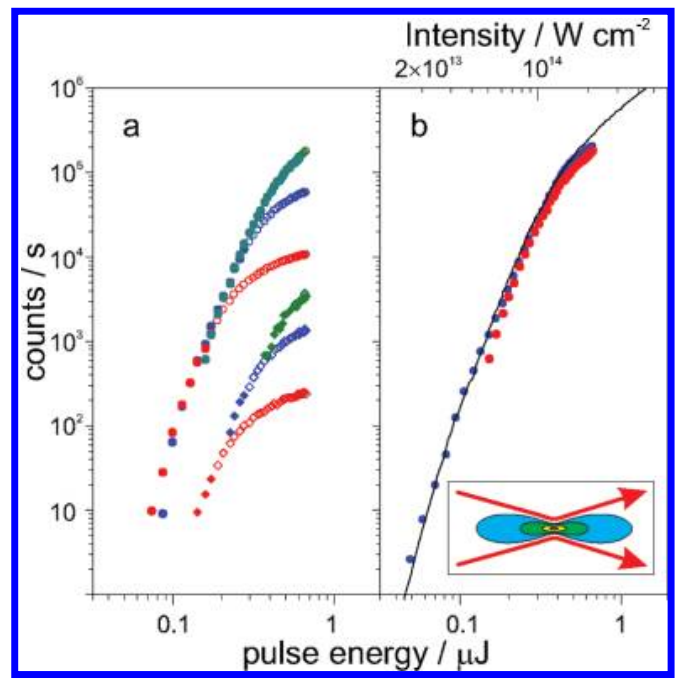

Figure 2. Concentration and laser repetition rate effects on the laser pulse energy dependence of Xe ionization: (a) normalized ion signal (counts/s) vs energy curves obtained at 725 (red), 72.5 (blue), and $7.25 \mathrm{ppm} \mathrm{Xe}$ (green). The circles plot the total $\mathrm{Xe}^{+}$signal and the diamonds the ${ }^{128} \mathrm{Xe}^{+}$signal. The laser repetition rate was $20 \mathrm{~Hz}$; (b) normalized ion signal vs energy curves obtained at $100 \mathrm{kHz}, 0.725$ ppm Xe (blue circles) and $20 \mathrm{~Hz}, 7.25 \mathrm{ppm}$ (red circles). The yields are normalized to $725 \mathrm{ppm}$ and $20 \mathrm{~Hz}$ in both panels. The solid line in part $b$ shows the yield calculated using intense field ionization theory $(-)$. The inset depicts a section through a Gaussian laser focus showing the volumes where the laser intensity is above 0.01 (blue), 0.05 (green), 0.3 (yellow), and 0.8 (red) times the maximum intensity.

imposed. They clearly are not, with the higher concentrations showing earlier saturation. The signal for the minor ${ }^{128} \mathrm{Xe}$ isotope also shows saturation, ruling out pile-up and demonstrating that the saturation is a total ion count per laser shot effect. Because the total unnormalized count rate was kept well below $10^{6} \mathrm{~s}^{-1}$ by operating the laser at $20 \mathrm{~Hz}$, we can also rule out saturation of the TDC data channel. We attribute this source saturation to space-charge effects that prevent electrons from escaping the ionization volume so that recombination occurs and reduces the ion yield. Inspection of the curves shows that saturation occurs at $\sim 1500$ counts $/ s$ under the focal conditions (microscope objective NA $=0.25$ ) used for these experiments. Space charge saturation is a per shot rather than a count rate effect. Dividing by the $20 \mathrm{~Hz}$ repetition rate we obtain a saturation limit of 75 detected ions per shot. If the laser is operated at $100 \mathrm{kHz}$, this implies a space-charge limit of $7.5 \times 10^{6}$ counts/s. This is above the MS total count rate capacity so that at this repetition rate source saturation is not a problem in the present experiments.

Figure $2 \mathrm{~b}$ confirms that there is a direct scaling with repetition rate. The absolute count rate for the $100 \mathrm{kHz}$ data was $10^{6} \mathrm{~s}^{-1}$ at the highest laser pulse energy. The coincidence with the $20 \mathrm{~Hz}$ results normalized to the repetition rate confirms that source saturation is not interfering at $100 \mathrm{kHz}$. Together with the scaling with concentration it also shows that the transmission of ions through the aperture into the mass spectrometer does not depend on ion density or ion current, at least below the source saturation limit. The $100 \mathrm{kHz}$ results in Figure $2 \mathrm{~b}$ were obtained using a concentration of Xe of 0.725 $\mathrm{ppm}$. At this concentration we obtained 1350 counts $/ \mathrm{s}$ in the ${ }^{124} \mathrm{Xe}$ mass peak. At natural abundance, ${ }^{124} \mathrm{Xe}$ is present at $0.09 \%$ making its concentration $650 \mathrm{ppt}$. This sets the limit of detection (LOD) at $1 \mathrm{ppt}$. At this sensitivity, the upper limit of 


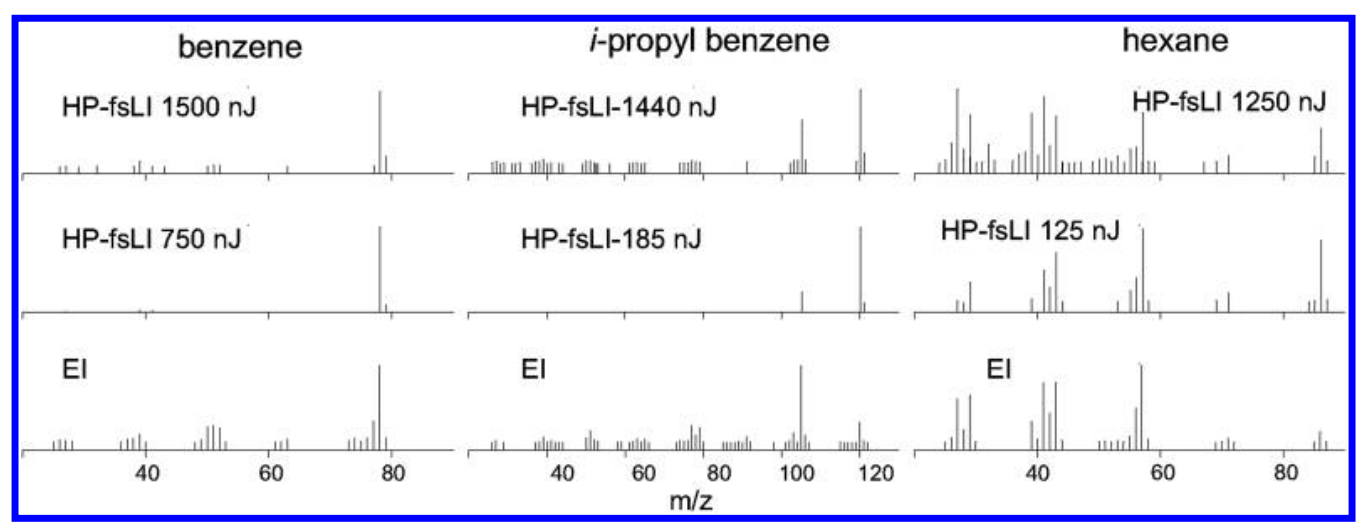

Figure 3. Mass spectra of benzene, iso-propyl benzene, and hexane obtained by high pressure femtosecond-laser ionization at the laser pulse energies indicated on each panel. Standard electron impact (EI) spectra from the NIST Chemistry WebBook ${ }^{12}$ are shown in the lower panels. The laser was focused with a 0.1 NA microscope objective and the pulse length was $40 \mathrm{fs}$ fwhm. Under these conditions, $120 \mathrm{~nJ}$ corresponds to a peak intensity of $10^{13} \mathrm{~W} \mathrm{~cm}^{-2}$. In the benzene spectrum at high intensity, the molecular ion peak $(\mathrm{m} / z=78)$ is saturated to make the small degree of fragmentation more visible.

concentration is $1 \mathrm{ppm}$, as determined by saturation effects. Higher concentrations can be accommodated at the expense of sensititvity by using lower laser intensities or repetition rates. A level of 1 ppt represents the LOD achieved with the HP-fsLI system in its current configuration.

We modeled the $\mathrm{Xe}^{+}$signal using intense field ionization theory to guide our understanding of how HP-fsLI depends on laser pulse parameters and focusing conditions. By comparison with the experiment, we confirm that we are capable of achieving $100 \%$ efficient ionization at the focus and can obtain a measurement of the ion collection efficiency of the mass spectrometer.

Figure $2 \mathrm{~b}$ is measured under conditions where the saturation effects discussed above are insignificant. Qualitatively the shape of the curve can be understood knowing that the nonresonant multiphoton ionization rate is highly nonlinear with laser intensity and that the intensity, $I$, varies over the laser focus, as depicted in the figure inset, according to

$$
I=\frac{I_{0}}{1+z^{2} / z_{0}^{2}} \exp \left(\frac{-2 r^{2}}{\omega_{0}^{2}\left(1+z^{2} / z_{0}^{2}\right)}\right)
$$

where $I_{0}$ is the peak intensity, $r$ is the radial distance from the center axis of the beam, and $z$ is the axial distance from the beam waist. $\omega_{0}$ is the radius at $1 / \mathrm{e}^{2}$ in intensity at the beam waist and $z_{0}=\pi \omega_{0}{ }^{2} / \lambda$, where $\lambda$ is the laser wavelength, is the Rayleigh range. At low intensities, the ion yield rises rapidly with intensity reflecting the high-order intensity dependence of the ionization process. At some point, the intensity is high enough so that the ionization probability at the beam waist effectively reaches 1 . This is the saturation intensity, $I_{\text {sat }}$. Although ionization at the waist is now saturated, the ion signal then continues to grow as the volume in which $I>I_{\text {sat }}$ grows as $I_{0}$ increases above $I_{\text {sat }}$, eventually reaching a regime where it becomes $\propto I^{1.5}$ reflecting the conical geometry of the beam away from the focus. The experimental curve in Figure 2 fits this expectation.

To model the ion signal, $S$, requires integration over the focal volume in space and over the laser pulse in time. For a Gaussian beam, the integration over space can be transformed to an integration over intensity leading to

$$
S=\alpha f V_{0} \mathcal{c} \int_{0}^{I_{0}}\left(1-\mathrm{e}^{-\int_{-\infty}^{\infty} W(I f(t)) \mathrm{d} t}\right) \frac{\sqrt{\frac{I_{0}}{I}-1}}{3 I}\left(\frac{I_{0}}{2 I}+1\right) \mathrm{d} I
$$

where $\alpha$ is the ion collection efficiency of the mass spectrometer, $f$ is the laser repetition rate, $c$ is the number density of analyte, and $V_{0}=2\left(\pi \omega_{0}^{2}\right)^{2} / \lambda$ is the focal volume. $(W(\operatorname{If}(t))$ is the ionization rate at intensity $\operatorname{If}(t)$ where $f(t)$ is the temporal pulse profile. For a square pulse of duration $\tau$, the integral $\int_{-\infty}^{\infty} W(I f(t)) \mathrm{d} t$ reduces to $W(I) \tau$.

The solid line in Figure 2 is obtained by solving eq 2 numerically with a Gaussian temporal profile with $f(t)=$ $\exp \left(-t^{2} / \tau^{2}\right)$ where $\tau$ is now the pulse half-width at $1 / \mathrm{e}$. Ionization rates are obtained using PPT theory. ${ }^{10}$ PPT theory is known to reproduce $\mathrm{Xe}$ ionization rates from the perturbative regime at low intensities to the tunneling regime at high intensities. ${ }^{11}$ In fitting the curve to the experimental result, we use the mass spectrometer ion collection efficiency, $\alpha$, and the beam radius, $\omega_{0}$, as fitting parameters. $\alpha$ is unknown and $\omega_{0}$ is hard to measure for a focal spot of the order of a micrometer. A low limit for $\omega_{0}$ can be estimated from the objective NA, but this assumes the optical system is aberration free and that the spatial mode of the beam is Gaussian. In intense field studies in high vacuum it is common to use $\mathrm{Xe}$ ionization yields to establish the intensity at a laser focus. ${ }^{7}$ Although two fitting parameters are used they are not highly sensitive to each other. $\alpha$ scales the signal vertically while $\omega_{0}$ influences the shape of the curve and the effective value of $I_{\text {sat }}$. The fit was obtained with $\omega_{0}=1.9 \mu \mathrm{m}$. The value of $\omega_{0}$ is not unreasonably higher than the theoretical diffraction limited value of $\omega_{0}, 1 \mu \mathrm{m}$. The value of $\alpha$ depends on how well the ion transmission of the mass spectrometer is optimized. The highest value of $\alpha$ that we have observed with the QStarXL using the HP-fsLI source is 0.03 . This implies that subpt LODs are attainable.

The model shows that we can fully understand high-pressure ionization yields using theory developed for isolated atoms. A $100 \%$ ionization efficiency is achievable, and the resulting ions can be effectively monitored using conventional mass spectrometry techniques.

lonization of Organics. Figure 3 shows mass spectra of benzene, iso-propyl benzene, and hexane obtained by HP-fsLI. These compounds were chosen because they are representative of three classes of compounds that are known to show different 
fragmentation behavior under femtosecond laser ionization at low pressure and because they are sufficiently volatile to make up known dilutions easily. In addition, hexane provides the opportunity to assess HP-fsLI of a class of compound that presents a difficult case for EI. The spectra were obtained from samples diluted in $\mathrm{He}$ at $\sim 100 \mathrm{ppb}$.

Figure 4a shows the dependence of the total ion signal of the organics on laser pulse energy and estimated intensity. The

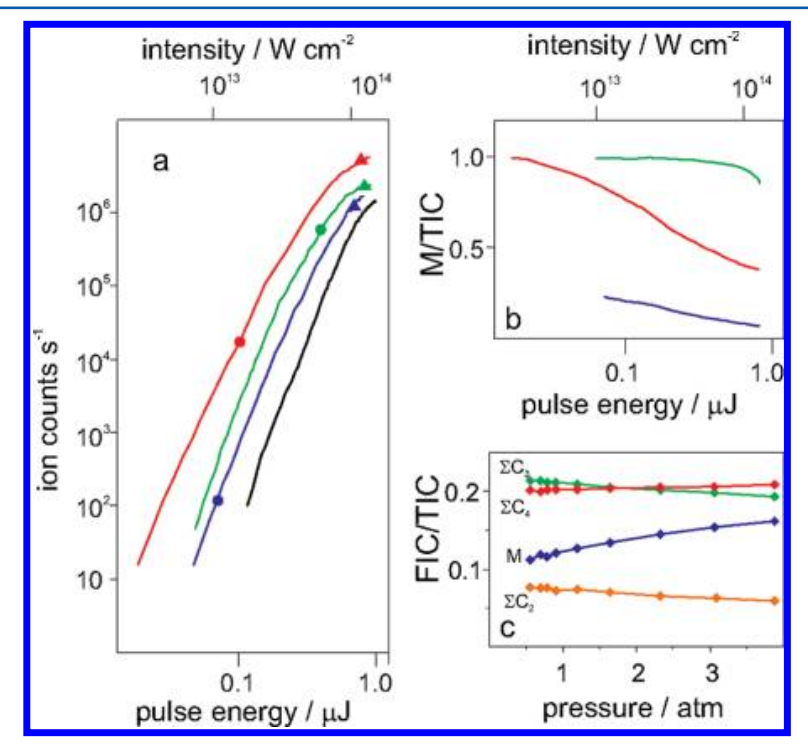

Figure 4. (a) Laser pulse energy dependence of the total ion signal obtained in the HP-fsLI of hexane (blue), benzene (green), iso-propyl benzene (red), and Xe (black). The curves are normalized to a concentration of $100 \mathrm{ppb}$ and were obtained with a 0.1 NA objective, a pulse width of $40 \mathrm{fs}$ fwhm, and a repetition rate of $100 \mathrm{kHz}$. The estimated peak laser intensity is given on the upper abscissa. The pulse energies at which the high and low energy mass spectra shown in Figure 3 were obtained are indicated by the triangle and circle symbols, respectively. (b) The degree of fragmentation, as indicated by the ratio of ion counts in the parent peak to the total ion counts (M/ TIC), as a function of laser pulse energy for the three organic molecules. Color coding is the same as in panel a. (c) Ratio of parent and selected fragment peak ion counts to the total ion count (FIC/ TIC) in the HP-fsLI of hexane as a function of pressure. The parent is shown with blue symbols and labeled $\mathrm{M}$. The sum of the counts in the fragments of stoichiometry $\mathrm{C}_{2} \mathrm{H}_{x}, \mathrm{C}_{3} \mathrm{H}_{x}$, and $\mathrm{C}_{4} \mathrm{H}_{x}$ are shown with orange, green, and red symbols and are labeled $\Sigma \mathrm{C}_{2}, \Sigma \mathrm{C}_{3}$, and $\Sigma \mathrm{C}_{4}$, respectively.

energy dependence of the ionization of all three molecules is very similar to that of $\mathrm{Xe}$ except that the curves are shifted to lower laser intensities. The extent of the shift is consistent with the IPs that are 10.13, 9.24, and $8.73 \mathrm{eV}$ for hexane, benzene, and iso-propyl benzene, respectively. The plot demonstrates that we can ionize at these representative organics with the same efficiency as we can Xe.

The pulse energies at which the spectra in Figure 3 were obtained are marked on Figure 4a to allow correlation of the fragmentation patterns with the ionization efficiency. Fragmentation is also addressed in Figure $4 \mathrm{~b}$ where the ratio of the ion count in the parent peak to the total ion count is shown as a function of laser energy.

At sufficiently low intensity, all three organic compounds show significantly reduced fragmentation compared to the EI spectra. For aromatics, as exemplified by benzene, the resistance to fragmentation is retained at high energy. Up to the saturation intensity all ion signal is in the parent peak. Even at the highest pulse energy used, well above the saturation intensity, $90 \%$ of the ion counts remain in the parent peak.

In contrast, hexane shows extensive fragmentation close to the saturation intensity that is similar to the EI pattern with the exception that more signal is retained in the parent peak. Only at relatively low intensities where the ionization probability is of the order of $10^{-3}-10^{-4}$ is the fragmentation substantially lower than in EI. Despite this propensity to fragment, $6 \%$ of the ion counts remain in the parent peak at intensities above $10^{14}$ $\mathrm{W} \mathrm{cm}{ }^{-2}$ where the ionzation probability reaches unity. The ability to observe parent peaks in the presence of severe fragmentation is partly a consequence of the irradiation geometry and is discussed in detail below in the section on geometry effects.

iso-Propyl benzene exemplifies an intermediate case. At the lowest intensities where the ionization probability is $10^{-4}$, the ion signal is concentrated in the parent peak. As the intensity increases it shows a single dominant fragmentation channel $\left(\mathrm{CH}_{3}\right.$ loss $)$ that persists until the ionization probability reaches 0.1. Above this the pattern is very similar to the EI spectrum, with the exception that, like hexane, the parent peak retains more strength.

\section{COLLISIONAL COOLING}

We speculated that an additional advantage of HP-fsLI is that collisional cooling might help reduce fragmentation in comparison to low-pressure fsLI. The collision frequency at atmospheric pressure is of the order of $5 \times 10^{9} \mathrm{~s}^{-1}$, making fragmentation channels with lifetimes of a nanosecond or greater open to quenching by collisional cooling.

Figure $4 c$ demonstrates that collisional cooling can indeed suppress some fragmentation in HP-fsLI. Hexane was chosen as the compound that showed the most fragmentation of the molecules studied here. The figure shows that the ratio of parent ion counts to the total ion count increases as the pressure in the ion source is increased from below 1 to 4 atm. The $\mathrm{C}_{4}$ envelope also shows a slight increase with pressure. At the same time the ratio of fragments of stoichiometry $\mathrm{C}_{2} \mathrm{H}_{x}$, $\mathrm{C}_{3} \mathrm{H}_{x}$ to the TIC decreases. We believe this behavior is a clear indication that collisional cooling is taking place.

In drawing this conclusion it is necessary to rule out the possibility that these results are skewed by a dependence of the mass spectrometer transmission on the pressure in the HP-fsLI ion source. Because the gas flow into the mass spectrometer changes with pressure in the ion source, we ensured that the pressure in $\mathrm{Q} 0$ was adjusted to a constant value by adjusting the makeup He flow. Under these conditions, we confirmed that the TIC scales linearly with the pressure in the ion source and, furthermore, that the mass spectrometer transmission function remains constant with pressure.

The modest cooling we observe for hexane is perhaps not too surprising considering that the time between collisions is in the range $0.1-0.25 \mathrm{~ns}$, that the collision gas is $\mathrm{He}$, and that hexane is a relatively small molecule. In small molecules, unimolecular dissociation rates can be relatively fast for a given internal energy. We might expect to see more collisional cooling in larger molecules with many more internal degrees of freedom but this remains to be seen. The pressure range in our setup was limited by the size of the aperture between the source and the mass spectrometer $(40 \mu \mathrm{m})$ combined with the pumping capacity of the mass spectrometer. Operation with a smaller aperture would allow much higher source pressures. For 
instance, a $10 \mu \mathrm{m}$ aperture would allow pressures up to $60 \mathrm{~atm}$ provided the ion source and gas delivery system can operate at this pressure.

\section{ION-MOLECULE CHEMISTRY}

In any atmospheric pressure ion source, it is essential to understand and control ion-molecule chemistry leading to chemical ionization, CI, especially at high analyte concentration. As can be seen in Figure 5, the HP-fsLI source is no

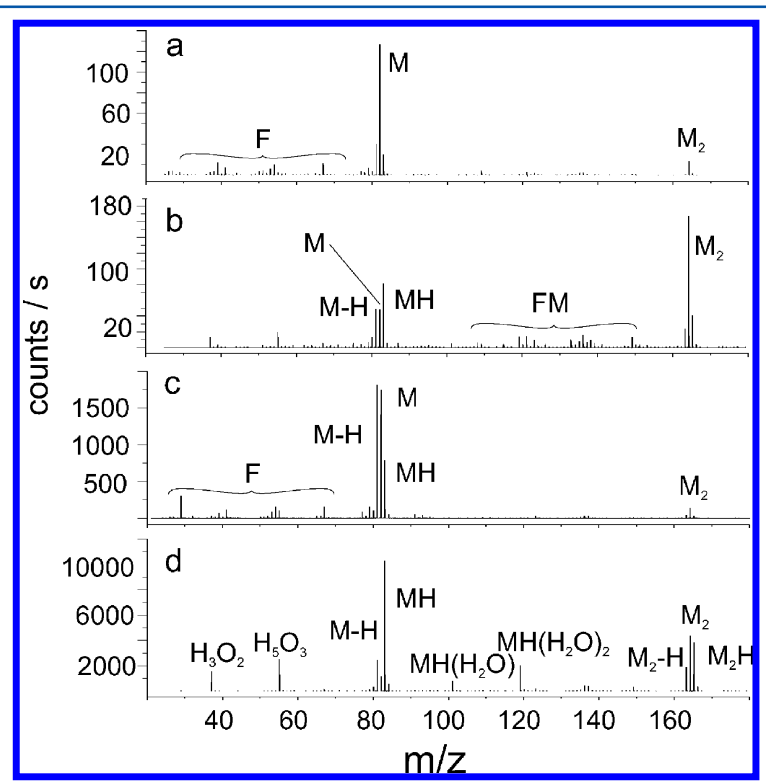

Figure 5. HP-fsLI mass spectra of cyclohexene $(0.7 \mathrm{ppm})$ in $\mathrm{He}$ containing $\mathrm{H}_{2} \mathrm{O}$ as an impurity. In panels a and $c$, the focus was within $50 \mu \mathrm{m}$ of the MS aperture; in panels $\mathrm{b}$ and $\mathrm{d}$, the focus was $1.1 \mathrm{~mm}$ away. In panels $a$ and $b$, the laser pulse energy was below the threshold for $\mathrm{H}_{2} \mathrm{O}$ ionization and in panels $\mathrm{c}$ and $\mathrm{d}$, it was above it. The peak labels correspond to $\mathrm{M}$, parent $\mathrm{C}_{6} \mathrm{H}_{10}{ }^{+}$ion; $\mathrm{M}_{2}$, the parent dimer ion; $\mathrm{MH}$, the protonated parent molecule; $\mathrm{M}-\mathrm{H}$, the $\mathrm{MH}^{+}$ion having lost a hydrogen molecule; F, collective fragment ions; FM, collective fragment-parent complex ions.

exception. The figure shows fsLI spectra of cyclohexene in a sample known to be contaminated with water. They were taken at laser pulse energies below and above the $\mathrm{H}_{2} \mathrm{O}$ ionization threshold and with the laser focus both close to the mass spectrometer aperture and $1.1 \mathrm{~mm}$ away. At low intensity and close to the aperture, the spectrum is dominated by the direct ionization and fragmentation of $\mathrm{C}_{6} \mathrm{H}_{10}$ driven by the laser pulse. The spectrum is largely similar to that obtained by fsLI at low pressure. However, even close to the aperture, ion-molecule chemistry is not entirely eliminated, as evidenced by the small peaks attributable to the $\left(\mathrm{C}_{6} \mathrm{H}_{10}\right)_{2}^{+}$dimer ion $\left(\mathrm{M}_{2}^{+}\right)$, the $\left(\mathrm{C}_{6} \mathrm{H}_{10}\right) \mathrm{H}^{+}$protonated parent ion $\left(\mathrm{MH}^{+}\right)$, and fragment produced by elimination of $\mathrm{H}_{2}$ from $\mathrm{MH}^{+}\left(\mathrm{M}-\mathrm{H}^{+}\right)$.

With the focus away from the aperture, the source behaves as a miniature flow reactor giving more time for ion molecule reactions to take place. This is shown in Figure 5b. As the focus is moved back from the aperture, the $\mathrm{M}_{2}^{+}, \mathrm{MH}^{+}$, and $\mathrm{M}-\mathrm{H}^{+}$ peaks are considerably enhanced. In addition, most of the signal strength in the direct fragment peaks is shifted into peaks that correspond to complexes of the fragment ions with the parent molecule. At low intensity, the protonation reactions are dominated by proton transfer from the parent and perhaps fragment ions to neutral $\mathrm{C}_{6} \mathrm{H}_{10}$ molecules.
At high intensity close to the aperture (Figure 5c), additional proton transfer occurs to enhance the $\mathrm{MH}^{+}$and $\mathrm{M}-\mathrm{H}^{+}$peaks. This is due to reaching the threshold to produce proton donors from water molecules that were present as an impurity in the gas feed in these experiments. The role of water is increasingly apparent as we move the focus away from the aperture at high intensity (Figure 5d) where the spectrum is dominated by the products of chemical ionization mainly driven by water ionization, including $\left(\mathrm{H}_{2} \mathrm{O}\right)_{n} \mathrm{H}^{+}$clusters, $\mathrm{MH}^{+}$, and $\mathrm{M}-\mathrm{H}^{+}$, water complexes of the protonated parent molecule $\left(\mathrm{MH}^{+}\left(\mathrm{H}_{2} \mathrm{O}\right)_{n}\right)$, and products of protonation of the $\left(\mathrm{C}_{6} \mathrm{H}_{10}\right)_{2}^{+}$ dimer ion $\left(\mathrm{M}_{2} \mathrm{H}^{+}\right.$and $\left.\mathrm{M}_{2}-\mathrm{H}^{+}\right)$. In separate experiments we have also observed ion-molecule charge transfer processes such as $\mathrm{Xe}+\mathrm{He}^{+} \rightarrow \mathrm{Xe}^{+}+\mathrm{He}$ and $\mathrm{Xe}^{+}+\mathrm{O}_{2} \rightarrow \mathrm{Xe}+\mathrm{O}_{2}^{+}$.

To study direct laser ionization, it is necessary to reduce CI to an insignificant level. This is achieved by keeping the focus as close to the aperture as possible, keeping the analyte concentration low enough to avoid CI reactions involving the parent molecule, and ensuring that the gas flow is free of contaminants such as water. These are the conditions we employed in the study of the HP-fsLI of the three organic molecules reported above.

While CI must be avoided to study direct laser ionization, it also presents an opportunity for a femtosecond laser driven approach to CI where the laser is used to deliberately create the proton donor. The deliberate addition of water will lead to the production of $\mathrm{H}_{3} \mathrm{O}^{+}$and higher hydronium clusters (depending on the $\mathrm{H}_{2} \mathrm{O}$ partial pressure) but, perhaps more unique as a CI agent, the addition of dry $\mathrm{H}_{2}$ at an appropriate pressure to avoid $\left(\mathrm{H}_{2}\right)_{n} \mathrm{H}^{+}$clusters can lead to $\mathrm{H}^{+}$. The proton itself can be regarded as the ultimate proton donor. Laser driven CI also provides a method of signal enhancement over direct ionization. Comparison of the total ion counts in panels $c$ and $\mathrm{d}$ of Figure 5 shows an order of magnitude increase when the focus is pulled back $1.1 \mathrm{~mm}$. This increase is consistent with the volume swept out by the ion packet as it moves from the focus to the sampling aperture.

Irradiation Geometry Effects, Process Optimization, and Observed Fragmentation Patterns. The laser intensity is determined by the pulse energy, the pulse width, and the focusing conditions. This raises several questions: What consideration needs to be given to optimizing these parameters? Should we be concerned that different analytes each require different conditions for efficient detection? Are there advantages of a high repetition rate low pulse energy laser over a lower repetition rate high pulse energy laser? How do observed fragmentation patterns relate to the distribution of intensities through the focal volume?

Simple consideration of eq 2 , where the ion signal depends on $V_{0}$, suggests that the laser should be focused to the largest possible waist size $\left.\left(V_{0}=2 \pi \omega_{0}^{2}\right)^{2} / \lambda\right)$ consistent with achieving an intensity, $I_{\text {sat }}$ where the ionization probability nears unity. Keeping the intensity below the Coulomb explosion (CE) limit, $I_{\mathrm{CE}}$, also would seem to argue for restricting $I_{0}$ and maximizing $V_{0}$. In intense field ionization, $I_{\mathrm{CE}}$ is the limit where the sudden removal of several electrons from a molecule results in drastic fragmentation driven by Coulomb repulsion. For organic molecules, $I_{\mathrm{CE}}$ is $2-3$ times $I_{\text {sat }}{ }^{7}$ implying that $I_{0}$ might need to be carefully controlled and perhaps adjusted for different molecules according to their $I_{\text {sat }}$. This would be inconvenient in an analytical chemistry context.

However, these simple considerations do not tell the whole story because the focused laser beam exposes molecules to all 
intensities below $I_{0}$ at some point in space. To follow the consequences, we consider a simple threshold model for ionization in which the rate for ionization is 0 below a threshold $I_{\text {th }}$ and infinite above it. Equation 2 then simplifies to

$$
S=\alpha f V_{0}\left(\frac{\gamma^{3}}{3}+2 \gamma-2 \arctan (\gamma)\right)=\alpha f V_{x}
$$

with $\gamma=\left(I_{0} / I_{\text {th }}-1\right)^{1 / 2} \cdot V_{x}$ is simply the volume in which $I>I_{\text {th. }}$. Figure 6 shows how $V_{x}$ varies with $\omega_{0}$ at a fixed pulse energy,

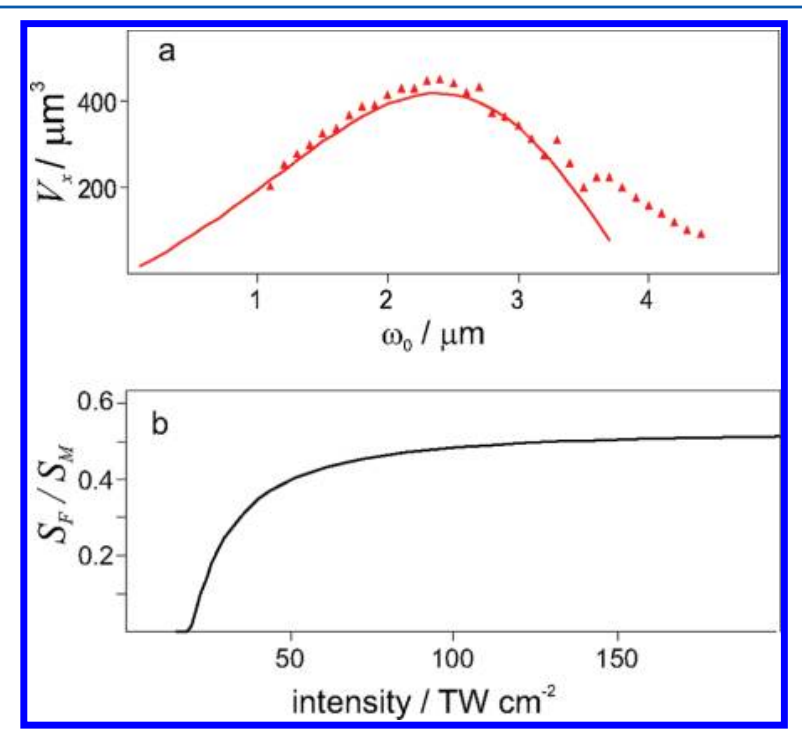

Figure 6. (a) Dependence of the effective ionization volume, $V_{x}$, on the beam waist radius, $\omega_{0}$, obtained from the threshold model, eq 3 (red line) and by numerical integration of eq 2 (points); (b) dependence of the ratio of the signal due to fragment ions to the signal due to molecular ions $\left(S_{\mathrm{F}} / S_{\mathrm{M}}\right)$ on laser peak intensity under the threshold model for an ionization threshold of $10 \mathrm{TW} \mathrm{cm}^{-2}$ and a fragmentation threshold of $20 \mathrm{TW} \mathrm{cm}^{-2}$.

$E_{0}$, and $I_{\mathrm{th}}$. The solid curve is from eq 3 , and the symbols are from a numerical solution of eq 2 using $W(I)$ values calculated for Xe using PPT theory. $I_{\text {th }}$ in the threshold model has been adjusted to $8.3 \times 10^{13} \mathrm{~W} \mathrm{~cm}^{-2}$ to fit the numerical prediction. The disagreement at large $\omega_{0}$ (low intensity) is an expected deficiency of the threshold model; however, the agreement is reasonable where $V_{x}$ reaches its maximum. It can be shown using the threshold model that the maximum occurs at a beam waist

$$
\omega_{\max }=\sqrt{\frac{0.13 E_{0}}{I_{\mathrm{th}} \tau}}
$$

This expression can be used to estimate the optimum focusing conditions from the laser parameters $E_{0}$ and $\tau$ and the threshold intensity $I_{\text {th }}$. At least for modest size organic molecules, with IPs in the range $8-10 \mathrm{eV}$, we can expect $I_{\text {th }}$ to be of the order of $40-80 \mathrm{TW} \mathrm{cm}^{-2}$. The required power of the focusing lens is available from the standard lens formula once the target $\omega_{\max }$ is established.

The threshold model can also be used to investigate the effect of increasing $E_{0}$ in the presence of fragmentation. This answers the questions of how careful one needs to be in keeping intensities below the Coulomb explosion limit discussed above and how we can continue to observe substantial parent peaks even when fragmentation is severe.
If undesirable fragmentation occurs at intensities above a threshold $I_{\mathrm{F}}$ while useful ionization occurs above a lower threshold, $I_{\mathrm{I}}$, we can use eq 3 to establish $V_{\mathrm{M}}$ the volume that contains useful ions as the difference between the volume above the ionization limit, $V_{\mathrm{J}}$, and the volume over the fragmentation limit, $V_{\mathrm{F}}$. Figure $6 \mathrm{~b}$ shows an example of the dependence of the ratio of undesirable fragment ions to useful ions as a function of $I_{0}$. Over the fragmentation threshold the fraction of fragment ions rises but it reaches a limiting value so that fragmentation can never dominate unless $I_{\mathrm{F}}$ is very close to $I_{\mathrm{M}}$. Inspection of eq 3 in the limit of $I_{0} \gg I_{x}$, where $\gamma \rightarrow\left(I_{0} / I_{x}\right)^{3 / 2}$, shows that this is expected, with $S_{\mathrm{F}} / S_{\mathrm{M}} \rightarrow 1 /\left\{\left(I_{\mathrm{F}} / I_{\mathrm{I}}\right)^{3 / 2}-1\right\}$. The curve in Figure $6 \mathrm{~b}$ was obtained with $I_{\mathrm{F}}=2 I_{\mathrm{I}}$ the lower range of the difference between ionization and Coulomb explosion thresholds. At intensities well above $I_{\mathrm{CE}}$ there are never less than twice the number of unexploded ions as CE fragments. As the small mass and multiply charged CE fragments can be discriminated against in the QStar mass spectrometer, it is reassuring to know that we can operate well above $I_{\mathrm{CE}}$ and still expect to observe substantial signal from molecular ions. This will be especially important when working with compounds whose fsLI has not been previously characterized.

\section{CONCLUSIONS}

We have shown that femtosecond laser ionization is an effective and by all indications universal ionization mechanism for gas phase molecules. High-pressure operation overcomes some of its seeming limitations, making it an efficient and highly sensitive ionization technique ideally suited to mass spectrometry. Even with a laser repetition rate of $100 \mathrm{kHz}$, we have demonstrated sensitivity of better than $1 \mathrm{ppt}$ and the sensitivity will scale linearly with the repetition rate for at least 3 orders of magnitude. In this respect we note that fiber-based femtosecond lasers are now commercially available that operate at up to $30 \mathrm{MHz}$ with pulse energies sufficient for fsLI. Fiber systems have the added advantage that they offer turnkey operation making them ideal for applications in fields where laser expertise is not readily available. Like EI, femtosecond laser ionization is generic. It seems likely that any molecule can be ionized. There are few requirements on laser wavelength provided the pulse is very short and the light wavelength is long enough that many photons are required for ionization. We have shown that the light intensity, the other seemingly critical parameter, does not need to be controlled very precisely at all.

Femtosecond laser ionization can be significantly softer compared to EI. Even for compounds that show significant fragmentation at the intensities required for near $100 \%$ ionization efficiency, almost fragment free ionization can be achieved by dropping the laser intensity by an order of magnitude.

HP-fsLI can also be used to seed chemical ionization. It is capable of producing a high, localized concentration of potentially interesting proton donor or charge transfer ions such as $\mathrm{H}^{+}$and $\mathrm{He}^{+}$that can be used to interrogate a larger volume than the laser ionization volume itself. In this respect HP-fsLI CI presents a method of further enhancing the sensitivity of the technique.

One requirement of HP-fsLI is that it uses He carrier gas to achieve its highest sensitivity. As such, we anticipate it will find direct application in the analysis of $\mathrm{He}$ gas streams from standard separation techniques such as gas chromatography and cryogenic gas exchange. In particular, we note that the gas throughput of our HP-fsLI mass spectrometer can be matched 
to the flow rates produced by capillary gas chromatographs. This promises efficient sampling for GC/MS using a highresolution mass spectrometer such as a high-pressure sampling QqTOF. We believe that this combination has the potential to provide new analytical capability that will justify the relatively high investment needed to introduce femtosecond laser technology to mass spectrometry. Although initial deployment may be in niche applications, we also believe that success in the analytical field will act as a driver to reduce the cost of suitable lasers.

\section{ASSOCIATED CONTENT}

\section{S Supporting Information}

Details of the analysis of interference due to carrier gas ionization and details of the characterization of pulse pile-up saturation. This material is available free of charge via the Internet at http://pubs.acs.org.

\section{AUTHOR INFORMATION}

\section{Corresponding Author}

*E-mail: David.Rayner@nrc-cnrc.gc.ca (D.M.R.); Alexandre. Loboda@absciex.com (A.V.L.).

\section{Notes}

The authors declare no competing financial interest.

\section{ACKNOWLEDGMENTS}

We gratefully acknowledge financial support from the Government of Ontario through the 2009 Premier's Discovery Award for Natural Science and Engineering to Paul Corkum.

\section{REFERENCES}

(1) Cole, R, Ed. Electrospray and MALDI Mass Spectrometry: Fundamentals, Instrumentation, Practicalities, and Biological Applications; Wiley: Hoboken, NJ, 2010.

(2) Takáts, Z.; Wiseman, J. M.; Gologan, B.; Cooks, R. G. Science 2004, 306, 471-473.

(3) Chernushevich, I.; Loboda, A.; Bruce, A.; Thomson, B. J. Mass Spectrom. 2001, 36, 849-865.

(4) Nemes, P.; Vertes, A. Anal. Chem. 2007, 79, 8098-8106.

(5) Ledingham, K.; Singhal, R. Int. J. Mass Spectrom. Ion Processes 1997, 163, 149-168.

(6) Beckey, H.-D. Field Ionization Mass Spectrometry; Pergamon Press: Oxford, U.K., 1971.

(7) Hankin, S. M.; Villeneuve, D. M.; Corkum, P. B.; Rayner, D. M. Phys. Rev. A 2001, 64, 013405/1-12.

(8) Hankin, S. M.; Villeneuve, D. M.; Corkum, P. B.; Rayner, D. M. Phvs. Rev. Lett. 2000, 84, 5082-5085.

(9) Larochelle, S. F. J.; Talebpour, A.; Chin, S. L. J. Phys. B 1998, 31, 1215-1224.

(10) Perelemov, A.; Popov, V.; Terent'ev, M. Sov. Phys. JETP 1966, 23, 924-934.

(11) Talebpour, A.; Yang, J.; Chin, S. L. Opt. Commun. 1999, 163, $29-32$.

(12) NIST Mass Spec Data Center. Stein, S. E., , Director. In NIST Chemistry WebBook, NIST Standard Reference Database Number 69; Linstrom, P., Mallard, W., Eds.; National Institute of Standards and Technology: Gaithersburg, MD, http://webbook.nist.gov, 2012. 